\title{
Article
}

\section{Air Pollution Is Associated with Poor Cognitive Function in Taiwanese Adults}

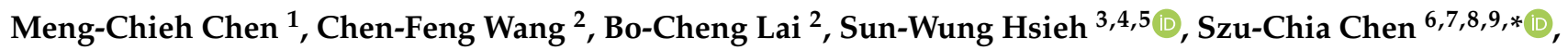 \\ Chih-Hsing Hung ${ }^{9,10,11}$ and Chao-Hung Kuo ${ }^{7,12}$
}

1 Department of General Medicine, Kaohsiung Medical University Hospital, Kaohsiung 807, Taiwan; b0103122@gmail.com

2 Institute of Electronics, National Chiao Tung University, Hsinchu 300, Taiwan; a114n.d425y@gmail.com (C.-F.W.); bclai@mail.nctu.edu.tw (B.-C.L.)

3 Department of Neurology, Kaohsiung Medical University Hospital, Kaohsiung Medical University, Kaohsiung 807, Taiwan; circle.6@yahoo.com.tw

4 Department of Neurology, Kaohsiung Municipal Siaogang Hospital, Kaohsiung Medical University, Kaohsiung 812, Taiwan

5 Neuroscience Research Center, Kaohsiung Medical University, Kaohsiung 807, Taiwan

6 Division of Nephrology, Department of Internal Medicine, Kaohsiung Medical University Hospital, Kaohsiung Medical University, Kaohsiung 807, Taiwan

7 Department of Internal Medicine, Kaohsiung Municipal Siaogang Hospital, Kaohsiung Medical University, Shan-Ming Rd., Hsiao-Kang Dist., Kaohsiung 812, Taiwan; kjh88kmu@gmail.com

8 Faculty of Medicine, College of Medicine, Kaohsiung Medical University, Kaohsiung 807, Taiwan

9 Research Center for Environmental Medicine, Kaohsiung Medical University, Kaohsiung 807, Taiwan; pedhung@gmail.com

10 Department of Pediatrics, Kaohsiung Medical University Hospital, Kaohsiung Medical University, Kaohsiung 807, Taiwan

11 Department of Pediatrics, Kaohsiung Municipal Siaogang Hospital, Kaohsiung Medical University, Kaohsiung 812, Taiwan

check for

updates

Citation: Chen, M.-C.; Wang, C.-F.; Lai, B.-C.; Hsieh, S.-W.; Chen, S.-C.;

Hung, C.-H.; Kuo, C.-H. Air Pollution

Is Associated with Poor Cognitive Function in Taiwanese Adults. Int. J. Environ. Res. Public Health 2021, 18,

316. https://doi.org/

10.3390/ijerph18010316

Received: 3 December 2020

Accepted: 30 December 2020

Published: 4 January 2021

Publisher's Note: MDPI stays neutral with regard to jurisdictional clai$\mathrm{ms}$ in published maps and institutional affiliations.

Copyright: $(\odot 2021$ by the authors. Licensee MDPI, Basel, Switzerland. This article is an open access article distributed under the terms and conditions of the Creative Commons Attribution (CC BY) license (https:// creativecommons.org/licenses/by/ $4.0 /)$.
12 Division of Gastroenterology, Department of Internal Medicine, Kaohsiung Medical University Hospital, Kaohsiung Medical University, Kaohsiung 807, Taiwan

* Correspondence: scarchenone@yahoo.com.tw; Tel.: +886-7-8036783 (ext. 3440); Fax: +886-7-8063346

Abstract: The issue of air pollution is gaining increasing attention worldwide, and mounting evidence has shown an association between air pollution and cognitive decline. The aim of this study was to investigate the relationships between air pollutants and cognitive impairment using the Mini-Mental State Exam (MMSE) and its sub-domains. In this study, we used data from the Taiwan Biobank combined with detailed daily data on air pollution. Cognitive function was assessed using the MMSE and its five subgroups of cognitive functioning. After multivariable linear regression analysis, a high level of particulate matter with an aerodynamic diameter of $\leq 2.5 \mu \mathrm{m}\left(\mathrm{PM}_{2.5}\right)$, low ozone $\left(\mathrm{O}_{3}\right)$, high carbon monoxide (CO), high sulfur dioxide $\left(\mathrm{SO}_{2}\right)$, high nitric oxide $(\mathrm{NO})$, high nitrogen dioxide $\left(\mathrm{NO}_{2}\right)$, and high nitrogen oxide $\left(\mathrm{NO}_{x}\right)$ were significantly associated with low total MMSE scores. Further, high $\mathrm{SO}_{2}$ and low $\mathrm{O}_{3}$ were significantly associated with low MMSE G1 scores. Low $\mathrm{O}_{3}$, high $\mathrm{CO}$, high $\mathrm{SO}_{2}$, high $\mathrm{NO}_{2}$, and high $\mathrm{NO}_{x}$ were significantly associated with low MMSE G4 scores, and high $\mathrm{PM}_{2.5}$, high particulate matter with an aerodynamic diameter of $\leq 10 \mu \mathrm{m}\left(\mathrm{PM}_{10}\right)$, high $\mathrm{SO}_{2}$, high $\mathrm{NO}_{2}$, and high $\mathrm{NO}_{\mathrm{x}}$ were significantly associated with low MMSE G5 scores. Our results showed that exposure to different air pollutants may lead to general cognitive decline and impairment of specific domains of cognitive functioning, and $\mathrm{O}_{3}$ may be a protective factor. These findings may be helpful in the development of policies regarding the regulation of air pollution.

Keywords: air pollutants; cognitive decline; mini-mental state exam; sub-domains

\section{Introduction}

Air pollution has become a serious concern worldwide. The World Health Organization (WHO) estimates that only one in ten people breathe air that does not contain 
pollutants exceeding the recommended limits. In the Asia-Pacific region, including Taiwan, air pollution is substantially more severe than in most developed countries, mostly due to rapid urbanization and industrialization with fewer emission regulations [1]. Air pollution has been shown to pose threats to health, and it has been linked to many diseases, including cardiovascular diseases, chronic obstructive pulmonary diseases, and even autoimmune diseases [2]. Emerging evidence has also shown associations between air pollution and cognitive decline and neurological disorders, such as Alzheimer's disease and Parkinson's disease [3], which will increase the economic burden on our aging society. Thus, there is a growing need to identify the relationships between air pollution and cognitive decline.

Various tools are available to measure cognitive function, such as the General Practitioner Assessment of Cognition, Mini-Cog ${ }^{\mathrm{TM}}$, and Mini-Mental State Exam (MMSE). Of these, the MMSE is a widely accepted tool that is easy to administer and divided into five subgroups to evaluate different aspects of cognitive functioning [4]. Most previous epidemiological studies investigating air pollution and cognitive impairment have focused on a limited number of pollutants, such as particulate matter (PM) and ozone $\left(\mathrm{O}_{3}\right)$. Experimental research has demonstrated that PM exposure can lead to diffuse accumulation of cerebral beta amyloid plaque, hyperphosphorylated tau pre-tangles, and peripheral systemic inflammation, which further result in the activation of microglial and astrocytes in the central nervous system (CNS). Moreover, neuroinflammation has been implicated as an important pathway in neurodegenerative disease [5]. $\mathrm{O}_{3}$, per se, is a strong oxidative pollutant, which elicits neurological injury by induction of release of free radicals, activation the generation of inflammatory cytokines and compromising the integrity of the blood-brain barrier. Despite most evidence showing its detrimental influence on cognition, a few epidemiological studies revealed protective effects at low concentrations of $\mathrm{O}_{3}$ exposure [6]. However, on the other hand, few studies have investigated other air pollutants, such as carbon monoxide $(\mathrm{CO})$, sulfur dioxide $\left(\mathrm{SO}_{2}\right)$, nitric oxide $(\mathrm{NO})$, nitrogen dioxide $\left(\mathrm{NO}_{2}\right)$, and nitrogen oxide $\left(\mathrm{NO}_{x}\right)[7,8]$, and the mechanisms on cognitive decline are still vague. Moreover, the results of relationships between these air pollutants and global cognitive function, and different trajectories of cognitive functioning, have been inconsistent [5].

In this study, we combined data from two databases, the Taiwan Biobank (TWB) and Taiwan Air Quality Monitoring Database (TAQMD). The TWB included 5000 registered individuals, and the TAQMD was comprised of detailed daily data on air pollution in Taiwan. Choe YM et al. evaluated the usefulness of subscores on the MMSE for predicting the progression of Alzheimer's disease dementia in individuals with mild cognitive impairment. They found that MMSE memory, orientation, and construction subscores, which are simple and readily available clinical measures, could provide useful information to predict Alzheimer's disease dementia progression in individuals with mild cognitive impairment, in practical clinical settings [9]. Therefore, we are very interested in knowing the effect of each air pollutant on its five subgroups of cognitive functioning. Thus, the aim of this study was to explore associations between the most well-known atmospheric air pollutants and cognitive impairment using the MMSE and its subdomains.

\section{Materials and Methods}

\subsection{Ethics Statement}

The Institutional Review Board on Biomedical Science Research, Academia Sinica, Taiwan, and the Ethics and Governance Council of the TWB, Taiwan granted ethical approval to the TWB. Each participant provided written informed consent, and the study was conducted according to institutional guidelines and the principles of the Declaration of Helsinki. In addition, this study was approved by the Institutional Review Board of Kaohsiung Medical University Hospital (KMUHIRB-E(I)-20180242).

\subsection{The Taiwan Biobank}

The TWB was established to record genomic and lifestyle data of Taiwanese residents, and it is currently the largest government-supported biobank in Taiwan $[10,11]$. The TWB 
is comprised of data of community-based volunteers aged 30 to 70 years with no history of cancer. A total of 5000 individuals who were registered in the TWB in April 2014 were enrolled in this study. All of the participants signed informed consent forms, gave blood samples, and underwent physical examinations. Data, including body height and weight, and body mass index (BMI) $\left(\mathrm{kg} / \mathrm{m}^{2}\right)$, were recorded. The participants were also asked to complete questionnaires about personal and lifestyle factors in face-to-face interviews with TWB researchers.

\subsection{Collection of Demographic, Medical and Laboratory Data}

Demographic data (age and sex), smoking history, medical history (diabetes mellitus (DM) and hypertension), examination findings (systolic (SBP) and diastolic blood pressures (DBP)) and laboratory data (fasting glucose, triglycerides, total cholesterol, hemoglobin, estimated glomerular filtration rate (eGFR) and uric acid) were recorded at baseline. EGFR was calculated using the four-variable Modification of Diet in Renal Disease equation [12].

\subsection{Evaluation of Cognitive Function}

We assessed the cognitive function of the subjects using the MMSE [4]. The participants completed MMSE questionnaires in face-to-face interviews with TWB researchers on the day of the enrollment. The MMSE is used as a screening tool for cognitive impairment, in which lower scores indicate that further evaluations are warranted. The MMSE contains five subscales: G1 orientation (score 0-10) (orientation to time and orientation to place), G2 registration (score 0-3), G3 attention and calculation (score 0-3), G4 recall (score 0-3), and G5 language (score 0-11) (including reading, repeat, naming, sentence, construction and obey). Total MMSE scores were calculated as the sum of each sub-score, with a maximum score of 30. Participants older than 60 years were invited to take the MMSE, and a total of 1054 participants who completed the MMSE questionnaire during the enrollment period were included in this study.

\subsection{Assessment of Air Pollutants}

The TAQMD was established by the Taiwan Environmental Protection Administration, Executive Yuan, and includes data on daily air pollutant concentrations at 74 ambient air quality monitoring stations around Taiwan. In this study, we linked the TAQMD and TWB by the location of the air quality monitoring stations and the area of residence of the participants. The residential address of each participant was used to estimate exposure to outdoor air pollution. The average concentrations of air pollutants, including PM with an aerodynamic diameter of $\leq 2.5 \mu \mathrm{m}\left(\mathrm{PM}_{2.5}\right)$, $\mathrm{PM}$ with an aerodynamic diameter of $\leq 10 \mu \mathrm{m}$ $\left(\mathrm{PM}_{10}\right), \mathrm{O}_{3}, \mathrm{CO}, \mathrm{SO}_{2}, \mathrm{NO}, \mathrm{NO}_{2}$, and $\mathrm{NO}_{\mathrm{x}}$ during each year were calculated, and average yearly data were determined in three steps: (1) the corresponding longitude and latitude of the address were obtained using Google geocoding; (2) the nearest air quality monitoring station was identified from an interpolation point; (3) data from the this station were filtered from the survey date to the previous year, and the average of each air pollution metric was calculated.

\subsection{Example of Nearest Neighbor Interpolation}

To illustrate our interpolation method and dataset distribution, we plotted the locations of the monitoring stations and participants as shown in the previous article [13].

\subsection{Statistical Analysis}

Data were expressed as mean \pm standard deviation, frequency (\%), or median (25th-75th percentile) for triglycerides. An MMSE cut-off score of 24 was used to classify the severity of cognitive impairment. Differences between groups were analyzed using the chi-square test for categorical variables and the independent $t$ test for continuous variables. Linear regression analysis was used to identify associations between each air pollutant and MMSE and subscales. A $p$ value of less than 0.05 was considered to indicate 
a statistically significant difference. Statistical analysis was performed using SPSS version 19.0 for Windows (SPSS Inc. Chicago, IL, USA).

\section{Results}

The mean age of the 1054 participants was $64.0 \pm 2.9$ years, and included 519 males and 535 females. The participants were stratified into two groups according to an MMSE score $\geq 24(\mathrm{n}=914,86.7 \%)$ or $<24(\mathrm{n}=140,13.3 \%)$. Comparisons of the clinical characteristics between these two groups are shown in Table 1. Compared to the participants with an MMSE score $\geq 24$, those with an MMSE score $<24$ were older, more predominantly female, had lower education years, low $\mathrm{O}_{3}$, lower total MMSE score, and lower score on each MMSE subscale.

Table 1. Comparison of clinical characteristics among participants according to total MMSE scores $\geq 24$ or $<24$.

\begin{tabular}{|c|c|c|c|c|}
\hline Characteristics & $\begin{array}{c}\text { All } \\
(\mathrm{n}=1054)\end{array}$ & $\begin{array}{c}\text { MMSE } \geq 24 \\
(n=914)\end{array}$ & $\begin{array}{c}\text { MMSE < } 24 \\
(n=140)\end{array}$ & $p$ \\
\hline Age (year) & $64.0 \pm 2.9$ & $63.9 \pm 2.9$ & $64.6 \pm 2.8$ & 0.015 \\
\hline Male gender $(\%)$ & 49.2 & 50.8 & 39.3 & 0.011 \\
\hline Smoking history (\%) & 24.8 & 25.5 & 20.0 & 0.161 \\
\hline Alcohol history (\%) & 5.7 & 5.8 & 5.0 & 0.704 \\
\hline $\mathrm{DM}(\%)$ & 11.5 & 11.4 & 12.1 & 0.792 \\
\hline Hypertension $(\%)$ & 24.2 & 24.4 & 22.9 & 0.692 \\
\hline Cerebrovascular disease (\%) & 1.1 & 1.2 & 0.7 & 1.000 \\
\hline Education (years) & $4.8 \pm 1.2$ & $5.0 \pm 1.1$ & $3.7 \pm 1.3$ & $<0.001$ \\
\hline Living alone (\%) & 9.2 & 9.4 & 7.9 & 0.554 \\
\hline Having job (\%) & 25.0 & 24.2 & 30.1 & 0.146 \\
\hline $\operatorname{BMI}\left(\mathrm{kg} / \mathrm{m}^{2}\right)$ & $24.4 \pm 3.0$ & $24.3 \pm 3.0$ & $24.8 \pm 3.1$ & 0.093 \\
\hline SBP $(\mathrm{mmHg})$ & $126.1 \pm 17.2$ & $125.9 \pm 17.1$ & $127.7 \pm 17.8$ & 0.251 \\
\hline $\mathrm{DBP}(\mathrm{mmHg})$ & $72.4 \pm 10.6$ & $72.5 \pm 10.7$ & $72.0 \pm 10.4$ & 0.617 \\
\hline \multicolumn{5}{|l|}{ Laboratory parameters } \\
\hline Fasting glucose (mg/dL) & $102.0 \pm 22.4$ & $101.6 \pm 22.0$ & $104.1 \pm 24.6$ & 0.229 \\
\hline Triglyceride (mg/dL) & $100(73-137)$ & $100(73-136.25)$ & $106.5(78-138.5)$ & 0.695 \\
\hline Total cholesterol (mg/dL) & $201.1 \pm 36.5$ & $201.3 \pm 36.9$ & $199.9 \pm 34.0$ & 0.678 \\
\hline Hemoglobin (g/dL) & $14.0 \pm 1.4$ & $14.1 \pm 1.4$ & $13.9 \pm 1.3$ & 0.383 \\
\hline $\mathrm{eGFR}\left(\mathrm{mL} / \mathrm{min} / 1.73 \mathrm{~m}^{2}\right)$ & $88.5 \pm 28.3$ & $88.2 \pm 28.3$ & $90.6 \pm 28.4$ & 0.354 \\
\hline Uric acid $(\mathrm{mg} / \mathrm{dL})$ & $5.7 \pm 1.4$ & $5.7 \pm 1.4$ & $5.7 \pm 1.4$ & 0.915 \\
\hline \multicolumn{5}{|l|}{ Air pollutants } \\
\hline $\mathrm{PM}_{2.5}\left(\mu \mathrm{g} / \mathrm{m}^{3}\right)$ & $35.2 \pm 11.5$ & $35.0 \pm 11.7$ & $36.5 \pm 10.3$ & 0.158 \\
\hline $\mathrm{PM}_{10}\left(\mu \mathrm{g} / \mathrm{m}^{3}\right)$ & $62.9 \pm 19.7$ & $62.7 \pm 19.9$ & $64.1 \pm 18.1$ & 0.436 \\
\hline $\mathrm{O}_{3}(\mathrm{ppb})$ & $30.9 \pm 4.1$ & $31.0 \pm 4.1$ & $30.1 \pm 3.8$ & 0.012 \\
\hline $\mathrm{CO}(\mathrm{ppm})$ & $0.45 \pm 0.21$ & $0.45 \pm 0.21$ & $0.47 \pm 0.20$ & 0.278 \\
\hline $\mathrm{SO}_{2}(\mathrm{ppb})$ & $3.5 \pm 1.4$ & $3.5 \pm 1.4$ & $3.7 \pm 1.2$ & 0.154 \\
\hline $\mathrm{NO}(\mathrm{ppb})$ & $4.3 \pm 4.4$ & $4.3 \pm 4.4$ & $4.7 \pm 4.5$ & 0.351 \\
\hline $\mathrm{NO}_{2}(\mathrm{ppb})$ & $14.6 \pm 6.8$ & $14.4 \pm 6.9$ & $15.5 \pm 6.0$ & 0.064 \\
\hline $\mathrm{NO}_{\mathrm{x}}(\mathrm{ppb})$ & $18.9 \pm 10.4$ & $18.7 \pm 10.5$ & $20.1 \pm 9.8$ & 0.131 \\
\hline \multicolumn{5}{|l|}{ MMSE } \\
\hline G1 (Orientation) & $9.5 \pm 0.8$ & $9.6 \pm 0.6$ & $8.6 \pm 1.3$ & $<0.001$ \\
\hline G2 (Registration) & $2.9 \pm 0.4$ & $2.9 \pm 0.3$ & $2.7 \pm 0.6$ & $<0.001$ \\
\hline G3 (Attention and Calculation) & $3.7 \pm 1.7$ & $4.1 \pm 1.5$ & $1.4 \pm 1.2$ & $<0.001$ \\
\hline G4 (Recall) & $2.2 \pm 0.9$ & $2.3 \pm 0.8$ & $1.3 \pm 1.1$ & $<0.001$ \\
\hline G5 (Language, construction, and obey) & $8.4 \pm 0.9$ & $8.5 \pm 0.7$ & $7.3 \pm 1.2$ & $<0.001$ \\
\hline MMSE total & $26.7 \pm 2.8$ & $27.5 \pm 1.9$ & $21.3 \pm 1.8$ & $<0.001$ \\
\hline
\end{tabular}

Abbreviations. MMSE, mini-mental state examination; DM, diabetes mellitus; BMI, body mass index; SBP, systolic blood pressure; DBP, diastolic blood pressure; eGFR, estimated glomerular filtration rate; $\mathrm{PM}_{2.5}$, particle with aerodynamic diameter of $2.5 \mu \mathrm{m}$ or less; $\mathrm{PM}_{10}$, particle with aerodynamic diameter of $10 \mu \mathrm{m}$ or less; $\mathrm{O}_{3}$, ozone; $\mathrm{CO}$, carbon monoxide; $\mathrm{SO}_{2}$; sulfur dioxide; $\mathrm{NO}$, nitric oxide; $\mathrm{NO}_{2}$, nitrogen dioxide; $\mathrm{NO}_{\mathrm{x}}$, nitrogen oxide.

Table 2 shows the determinants of total MMSE score in the study participants using multivariable linear regression analysis. Old age (unstandardized coefficient $\beta,-0.006$; $p=0.009$ ), female (unstandardized coefficient $\beta, 0.534 ; p=0.002$ ), low education years 
(unstandardized coefficient $\beta, 0.950 ; p=0.039$ ), high BMI (unstandardized coefficient $\beta,-0.092 ; p=0.001$ ), low $\mathrm{O}_{3}$ (unstandardized coefficient $\beta, 0.047 ; p<0.029$ ), and high $\mathrm{SO}_{2}$ (unstandardized coefficient $\beta,-0.145 ; p=0.022$ ) were associated with low total MMSE score.

Table 2. Association of clinical characteristic and air pollutants with total MMSE scores using univariable linear regression analysis.

\begin{tabular}{|c|c|c|}
\hline \multirow{2}{*}{ Characteristics } & \multicolumn{2}{|c|}{ Univariable } \\
\hline & Unstandardized Coefficient $\beta$ (95\% CI) & $p$ \\
\hline Age (per 1 year) & $-0.006(-0.011,-0.002)$ & 0.009 \\
\hline Male gender (vs. female) & $0.534(0.194,0.874)$ & 0.002 \\
\hline Smoking history & $0.340(-0.055,0.735)$ & 0.092 \\
\hline Alcohol history & $0.355(-0.381,1.092)$ & 0.344 \\
\hline $\mathrm{DM}$ & $-0.359(-0.895,0.176)$ & 0.188 \\
\hline Hypertension & $-0.104(-0.502,0.295)$ & 0.610 \\
\hline Cerebrovascular disease & $0.137(-1.473,1.746)$ & 0.868 \\
\hline Education (per 1 years) & $0.950(0.825,1.075)$ & $<0.001$ \\
\hline Living alone & $-0.012(-0.058,0.034)$ & 0.619 \\
\hline Having job & $-0.325(-0.716,0.067)$ & 0.104 \\
\hline BMI (per $\left.1 \mathrm{~kg} / \mathrm{m}^{2}\right)$ & $-0.092(-0.148,-0.037)$ & 0.001 \\
\hline SBP (per $1 \mathrm{mmHg}$ ) & $-0.003(-0.013,0.007)$ & 0.547 \\
\hline DBP (per $1 \mathrm{mmHg}$ ) & $0.011(-0.005,0.027)$ & 0.192 \\
\hline \multicolumn{3}{|l|}{ Laboratory parameters } \\
\hline Fasting glucose (per 1 mg/dL) & $-0.007(-0.015,0.001)$ & 0.069 \\
\hline Triglyceride (log per $1 \mathrm{mg} / \mathrm{dL}$ ) & $-0.539(-1.354,0.276)$ & 0.195 \\
\hline Total cholesterol (per $1 \mathrm{mg} / \mathrm{dL}$ ) & $0.002(-0.002,0.007)$ & 0.352 \\
\hline Hemoglobin (per $1 \mathrm{~g} / \mathrm{dL}$ ) & $0.069(-0.054,0.193)$ & 0.272 \\
\hline eGFR $\left(\right.$ per $1 \mathrm{~mL} / \mathrm{min} / 1.73 \mathrm{~m}^{2}$ ) & $-0.004(-0.010,0.002)$ & 0.171 \\
\hline Uric acid (per $1 \mathrm{mg} / \mathrm{dL}$ ) & $-0.025(-0.147,0.097)$ & 0.688 \\
\hline \multicolumn{3}{|l|}{ Air pollutants } \\
\hline $\mathrm{PM}_{2.5}\left(\right.$ per $\left.1 \mu \mathrm{g} / \mathrm{m}^{3}\right)$ & $-0.010(-0.025,0.005)$ & 0.182 \\
\hline $\mathrm{PM}_{10}\left(\right.$ per $\left.1 \mu \mathrm{g} / \mathrm{m}^{3}\right)$ & $-0.004(-0.013,0.004)$ & 0.328 \\
\hline $\mathrm{O}_{3}($ per $1 \mathrm{ppb})$ & $0.047(0.005,0.089)$ & 0.029 \\
\hline $\mathrm{CO}$ (per 1 ppm) & $-0.380(-1.222,0.462)$ & 0.376 \\
\hline $\mathrm{SO}_{2}($ per $1 \mathrm{ppb})$ & $-0.145(-0.270,-0.021)$ & 0.022 \\
\hline $\mathrm{NO}($ per 1 ppb) & $-0.016(-0.055,0.023)$ & 0.426 \\
\hline $\mathrm{NO}_{2}($ per $1 \mathrm{ppb})$ & $-0.023(-0.048,0.003)$ & 0.080 \\
\hline $\mathrm{NO}_{\mathrm{x}}($ per $1 \mathrm{ppb})$ & $-0.013(-0.029,0.004)$ & 0.135 \\
\hline
\end{tabular}

Values expressed as unstandardized coefficient $\beta$ and 95\% confidence interval (CI). Abbreviations are the same as in Table 1.

\subsection{Correlations between Air Pollutants and Total MMSE Scores}

Table 3 shows the determinants of total MMSE score in the study participants using multivariable linear regression analysis after adjusting for living alone, being employed, age, sex, smoking and alcohol history, DM, hypertension, cerebrovascular disease, BMI, SBP, DBP, fasting glucose, log triglycerides, total cholesterol, hemoglobin, eGFR, uric acid and each air pollutant. High $\mathrm{PM}_{2.5}$ (unstandardized coefficient $\beta,-0.014 ; p=0.039$ ), low $\mathrm{O}_{3}$ (unstandardized coefficient $\beta, 0.078 ; p<0.001$ ), high $\mathrm{CO}$ (unstandardized coefficient $\beta,-1.133 ; p=0.005$ ), high $\mathrm{SO}_{2}$ (unstandardized coefficient $\beta,-0.227 ; p<0.001$ ), high $\mathrm{NO}$ (unstandardized coefficient $\beta,-0.042 ; p=0.022$ ), high $\mathrm{NO}_{2}$ (unstandardized coefficient $\beta$, $-0.043 ; p<0.001$ ), and high $\mathrm{NO}_{\mathbf{x}}$ (unstandardized coefficient $\beta,-0.026 ; p=0.001$ ) were significantly associated with low total MMSE score, whereas $\mathrm{PM}_{10}$ was not significantly associated with total MMSE score. 
Table 3. Association of air pollutants with total MMSE scores using multivariable linear regression analysis.

\begin{tabular}{ccc}
\hline \multirow{2}{*}{ Air Pollutants } & \multicolumn{2}{c}{ Multivariable } \\
\cline { 2 - 3 } & Unstandardized Coefficient $\boldsymbol{\beta} \mathbf{( 9 5 \%}$ CI) & $p$ \\
\hline $\mathrm{PM}_{2.5}\left(\right.$ per $\left.1 \mu \mathrm{g} / \mathrm{m}^{3}\right)$ & $-0.014(-0.028,0)$ & 0.039 \\
$\mathrm{PM}_{10}$ (per $\left.1 \mu \mathrm{g} / \mathrm{m}^{3}\right)$ & $-0.006(-0.015,0.002)$ & 0.131 \\
$\mathrm{O}_{3}$ (per 1 ppb) & $0.078(0.039,0.117)$ & $<0.001$ \\
$\mathrm{CO}$ (per 1 ppm) & $-1.133(-1.915,-0.351)$ & 0.005 \\
$\mathrm{SO}_{2}$ (per 1 ppb) & $-0.227(-0.342,-0.111)$ & $<0.001$ \\
$\mathrm{NO}$ (per 1 ppb) & $-0.042(-0.079,-0.006)$ & 0.022 \\
$\mathrm{NO}_{2}$ (per 1 ppb) & $-0.043(-0.067,-0.019)$ & $<0.001$ \\
$\mathrm{NO}_{\mathbf{x}}$ (per 1 ppb) & $-0.026(-0.042,-0.011)$ & 0.001 \\
\hline Values expressed as unstandardized coefficient $\beta$ and 95\% confidence interval (CI). Abbreviations are the same \\
as in Table 1. Multivariable model: adjusted for age, sex, smoking and alcohol history, DM, hypertension, \\
cerebrovascular disease, life style with living alone and having job, BMI, SBP, DBP, fasting glucose, log triglyceride, \\
total cholesterol, hemoglobin, eGFR, uric acid, and each air pollutants.
\end{tabular}

\subsection{Correlations between Air Pollutants and Each MMSE Subscore}

Table 4 shows the determinants of MMSE G1 (orientation) score in the study participants using multivariable linear regression analysis. The results showed that high $\mathrm{SO}_{2}$ (unstandardized coefficient $\beta,-0.044 ; p=0.015$ ), and low $\mathrm{O}_{3}$ (unstandardized coefficient $\beta, 0.014 ; p=0.020$ ) were significantly associated with a low MMSE G1 score.

Table 4. Association of air pollutants with MMSE G1 (orientation) using multivariable linear regression analysis.

\begin{tabular}{ccc}
\hline \multirow{2}{*}{ Air Pollutants } & \multicolumn{1}{c}{ Multivariable } \\
\cline { 2 - 3 } & Unstandardized Coefficient $\boldsymbol{\beta} \mathbf{( 9 5 \% ~ C I )}$ & $\boldsymbol{p}$ \\
\hline $\mathrm{PM}_{2.5}\left(\right.$ per $\left.1 \mu \mathrm{g} / \mathrm{m}^{3}\right)$ & $-0.001(-0.006,0.003)$ & 0.532 \\
$\mathrm{PM}_{10}$ (per 1 $\left.\mu \mathrm{g} / \mathrm{m}^{3}\right)$ & $0(-0.003,0.002)$ & 0.757 \\
$\mathrm{O}_{3}$ (per 1 ppb) & $0.014(0.002,0.026)$ & 0.020 \\
$\mathrm{CO}$ (per 1 ppm) & $-0.175(-0.413,0.063)$ & 0.150 \\
$\mathrm{SO}$ (per 1 ppb) & $-0.044(-0.079,-0.009)$ & 0.015 \\
$\mathrm{NO}$ (per 1 ppb) & $-0.010(-0.021,0.001)$ & 0.082 \\
$\mathrm{NO}_{2}$ (per 1 ppb) & $-0.004(-0.012,0.003)$ & 0.232 \\
$\mathrm{NO}_{\mathrm{x}}$ (per 1 ppb) & $-0.004(-0.008,0.001)$ & 0.128 \\
\hline
\end{tabular}

Values expressed as unstandardized coefficient $\beta$ and $95 \%$ confidence interval (CI). Abbreviations are the same as in Table 1. Multivariable model: adjusted for age, sex, smoking and alcohol history, DM, hypertension, cerebrovascular disease, life style with living alone and having job, BMI, SBP, DBP, fasting glucose, log triglyceride, total cholesterol, hemoglobin, eGFR, uric acid, and each air pollutants.

Table 5 shows the determinants of MMSE G4 (recall) score in the study participants using multivariable linear regression analysis. The results showed that low $\mathrm{O}_{3}$ (unstandardized coefficient $\beta, 0.016 ; p=0.023$ ), high $C O$ (unstandardized coefficient $\beta,-0.284$; $p=0.047$ ), high $\mathrm{SO}_{2}$ (unstandardized coefficient $\beta,-0.065 ; p=0.002$ ), high $\mathrm{NO}_{2}$ (unstandardized coefficient $\beta,-0.010 ; p=0.019$ ) and high $\mathrm{NO}_{\mathrm{x}}$ (unstandardized coefficient $\beta$, $-0.006 ; p=0.026$ ) were significantly associated with a low MMSE G4 score.

Table 6 demonstrates the determinants of MMSE G5 (language) score in the study participants using multivariable linear regression analysis. The results showed that high $\mathrm{PM}_{2.5}$ (unstandardized coefficient $\beta,-0.005 ; p=0.022$ ), high $\mathrm{PM}_{10}$ (unstandardized coefficient $\beta,-0.003 ; p=0.013$ ), high $\mathrm{SO}_{2}$ (unstandardized coefficient $\beta,-0.057 ; p=0.003$ ), high $\mathrm{NO}_{2}$ (unstandardized coefficient $\beta,-0.011 ; p=0.006$ ), and high $\mathrm{NO}_{\mathrm{x}}$ (unstandardized coefficient $\beta,-0.006 ; p=0.020$ ) were significantly associated with a low MMSE G5 score. 
Table 5. Association of air pollutants with MMSE G4 (recall) using multivariable linear regression analysis.

\begin{tabular}{ccc}
\hline \multirow{2}{*}{ Air Pollutants } & \multicolumn{1}{c}{ Multivariable } \\
\cline { 2 - 3 } & Unstandardized Coefficient $\boldsymbol{\beta} \mathbf{( 9 5 \% \mathbf { C I } )}$ & $\boldsymbol{p}$ \\
\hline $\mathrm{PM}_{2.5}\left(\right.$ per $\left.1 \mu \mathrm{g} / \mathrm{m}^{3}\right)$ & $-0.002(-0.007,0.003)$ & 0.375 \\
$\mathrm{PM}_{10}$ (per 1 $\left.\mu \mathrm{g} / \mathrm{m}^{3}\right)$ & $-0.002(-0.005,0.001)$ & 0.178 \\
$\mathrm{O}_{3}$ (per 1 ppb) & $0.016(0.002,0.030)$ & 0.023 \\
$\mathrm{CO}$ (per 1 ppm) & $-0.284(-0.565,-0.003)$ & 0.047 \\
$\mathrm{SO}$ (per 1 ppb) & $-0.065(-0.107,-0.024)$ & 0.002 \\
$\mathrm{NO}$ (per 1 ppb) & $-0.010(-0.023,0.003)$ & 0.121 \\
$\mathrm{NO}_{2}$ (per 1 ppb) & $-0.010(-0.019,-0.002)$ & 0.019 \\
$\mathrm{NO}_{\mathrm{x}}$ (per 1 ppb) & $-0.006(-0.012,0)$ & 0.026 \\
\hline
\end{tabular}

Values expressed as unstandardized coefficient $\beta$ and $95 \%$ confidence interval (CI). Abbreviations are the same as in Table 1. Multivariable model: adjusted for age, sex, smoking and alcohol history, DM, hypertension, cerebrovascular disease, life style with living alone and having job, BMI, SBP, DBP, fasting glucose, log triglyceride, total cholesterol, hemoglobin, eGFR, uric acid, and each air pollutants.

Table 6. Association of air pollutants with MMSE G5 (language, construction, and obey) using multivariable linear regression analysis.

\begin{tabular}{ccc}
\hline \multirow{2}{*}{ Air Pollutants } & \multicolumn{1}{c}{ Multivariable } \\
\cline { 2 - 3 } & Unstandardized Coefficient $\beta \mathbf{~ ( 9 5 \% ~ C I ) ~}$ & $p$ \\
\hline $\mathrm{PM}_{2.5}\left(\right.$ per $\left.1 \mu \mathrm{g} / \mathrm{m}^{3}\right)$ & $-0.005(-0.010,0)$ & 0.022 \\
$\mathrm{PM}_{10}$ (per 1 $\left.\mu \mathrm{g} / \mathrm{m}^{3}\right)$ & $-0.003(-0.006,0)$ & 0.013 \\
$\mathrm{O}_{3}$ (per 1 ppb) & $0.012(0,0.025)$ & 0.058 \\
$\mathrm{CO}$ (per 1 ppm) & $-0.247(-0.504,0.011)$ & 0.061 \\
$\mathrm{SO}$ (per 1 ppb) & $-0.057(-0.095,-0.019)$ & 0.003 \\
$\mathrm{NO}$ (per 1 ppb) & $-0.008(-0.020,0.004)$ & 0.196 \\
$\mathrm{NO}_{2}$ (per 1 ppb) & $-0.011(-0.019,-0.003)$ & 0.006 \\
$\mathrm{NO}_{\mathrm{x}}$ (per 1 ppb) & $-0.006(-0.011,0)$ & 0.020 \\
\hline
\end{tabular}

Values expressed as unstandardized coefficient $\beta$ and 95\% confidence interval (CI). Abbreviations are the same as in Table 1. Multivariable model: adjusted for age, sex, smoking and alcohol history, DM, hypertension, cerebrovascular disease, life style with living alone and having job, BMI, SBP, DBP, fasting glucose, log triglyceride, total cholesterol, hemoglobin, eGFR, uric acid, and each air pollutants.

We also analyzed correlations between air pollutants and MMSE G2 (registration) and G3 (attention and calculation) scores. However, no air pollutants were significantly associated with these scores, except for low $\mathrm{O}_{3}$ (unstandardized coefficient $\beta, 0.033 ; p=0.011$ ), which was associated with a low MMSE G3 score.

\section{Discussion}

In this study of 1054 participants registered in the TWB from around Taiwan, we observed that high $\mathrm{PM}_{2.5}, \mathrm{CO}, \mathrm{SO}_{2}, \mathrm{NO}, \mathrm{NO}_{2}$, and $\mathrm{NO}_{\mathrm{x}}$, and low $\mathrm{O}_{3}$ were correlated with low total MMSE score. We further found that these pollutants were separately correlated with different MMSE subscores.

The first important finding of this study is that high $\mathrm{PM}_{2.5}$ was associated with low total MMSE and MMSE G5 (language) scores, and that high PM $_{10}$ was also related with a low MMSE G5 score. Increasing epidemiological evidence has shown the effect of $\mathrm{PM}_{2.5}$ on worsening cognitive function [13-16]. In one prospective cohort study, Wang et al. reported the adverse effect of long-term exposure to $\mathrm{PM}_{2.5}$ on worsening cognitive function [17]. In addition, Calderón-Garcidueñas also reported that exposure to $\mathrm{PM}_{2.5}$ could also cause deficits in multiple domains of cognitive function, including executive, memory, visuospatial, and language functions in young adults [18]. The primary mechanism by which PM damages the cardiovascular and pulmonary systems is mainly by inducing both local and systemic oxidative stress and inflammation [19]; however, the detailed mechanism by 
which PM damages the CNS has yet to be elucidated. Once inhaled, PM can cause direct cell damage via free radicals and stimulate the release of inflammatory cytokines, which can then induce the release of cytokines and reactive oxygen species in the brain. This then leads to a cycle of neuroinflammation and oxidative damage, and finally injury to the CNS [20-22]. PM can also make the CNS more susceptible to systemic inflammation by destroying the integrity of the blood-brain barrier (BBB) after directly entering the brain via the olfactory nerve and altering microbial balance in the gastrointestinal tract, which indirectly leads to an imbalance of immunity in the CNS [23]. With regards to pathological changes in the brain, growing evidence supports that airborne $\mathrm{PM}_{2.5}$ accelerates the accumulation of cerebral $\beta$-amyloid and tau hyper-phosphorylation [24,25], which are typical in the development of Alzheimer disease. From a macroscopic perspective, a previous study demonstrated an association between $\mathrm{PM}_{2.5}$ and smaller hippocampal volume [26]. Taken together, these findings indicate that exposure to $\mathrm{PM}_{2.5}$ is a risk for the development of cognitive decline and CNS damage. However, results of the association between cognitive decline and the coarser particles of $\mathrm{PM}_{10}$, with an aerodynamic diameter of 2.5 to $10 \mathrm{~mm}$, have been inconsistent [27-30]. Shin et al. proposed that the difference between $\mathrm{PM}_{2.5}$ and $\mathrm{PM}_{10}$ is the ability to more readily cross the BBB and cause more systematic inflammation [16], although numerous studies have suggested that nanoparticles, some of which are larger than $\mathrm{PM}_{10}$, can reach the CNS via numerous routes, including inhalation through the lungs, direct capture by the olfactory nerve, digestion into the gastrointestinal tract, or cutaneous pathways [22]. These findings may imply that exposure to PM pollutants can lead to cognitive decline.

The second important finding of this study is that high $\mathrm{O}_{3}$ was associated with high total MMSE score and G1, G3, G4 subscores, implying the possible neuroprotective effect of $\mathrm{O}_{3}$ on cognitive function. Previous experimental and epidemiological studies have identified that $\mathrm{O}_{3}$, a strong oxidative pollutant and, therefore, potentially toxic to humans, has detrimental effects on the CNS $[13,14,18,30]$. Synergistic and dose effects with PM have also been reported [13,31]. Inhaled $\mathrm{O}_{3}$ not only directly damages lung function, but also cause systemic effects via the generation of free radicals. Oxidative stress then further increases the expression of inducible $\mathrm{NO}$ and subsequent cellular response, which leads to increases in leukotrienes, prostaglandins, tumor necrosis factor, interleukin-1, interleukin-6, and interleukin-8. Through the active transport of endothelial cells adjacent to the BBB or via other proposed methods of entry into the $\mathrm{CNS}$, these molecules can affect astrocytes and then compromise the integrity of the $\mathrm{BBB}$, and this process can be accelerated by free radicals caused by exposure to $\mathrm{O}_{3}$. This vicious cycle has been suggested to lead to the development of neurological disorders and cognitive impairment [32]. However, in a study in Korea, Shin et al. found a positive association between $\mathrm{O}_{3}$ and a variety of measures for cognition including MMSE Korea child scores, digit-forward span scores, word list recall, recall storage, and frontal assessment battery scores [16]. Similar results were also found in a cross-sectional study by Gatto et al. in Los Angeles [30] and a cohort study by Carey et al. in London [33]. The discrepancy may be due to a dose-effect, as the positive findings were linked to similar low concentrations of $\mathrm{O}_{3}$ exposure. Although exposure to toxic levels of $\mathrm{O}_{3}$ as an air pollutant has been considered to induce systemic inflammation and damage, exposure to low $\mathrm{O}_{3}$ concentrations has been used for medical purposes via activating Nrf2, which can enhance the activity of antioxidant enzymes such as superoxide dismutase and catalase [34]. This may provide a mechanism by which $\mathrm{O}_{3}$ has a positive effect on the CNS in contrast to the other reported detrimental effects. Further experimental and epidemiological studies are needed to investigate the relationships between $\mathrm{O}_{3}$ as an air pollutant and the CNS to fill this gap in knowledge.

The third important finding of this study is that high CO was correlated with low total MMSE score and recall domain scores, suggesting the detrimental effect on cognitive function. Chang et al. demonstrated the negative effect of $\mathrm{CO}$ on cognitive performance [6], and Shin et al. also reported associations between increased risks of poor global cognition, low attention, and executive function and CO exposure [16]. CO has a 200-fold greater 
affinity for hemoglobin than oxygen, and the decreased binding capacity of oxygen can lead to a reduction in the amount of oxygen reaching the periphery tissue, including the brain. Acute exposure to $\mathrm{CO}$ can cause tissue hypoxia-ischemia, cardiac compromise, and shock, which can further worsen hypoxia of the CNS and subsequently cause neurological damage $[35,36]$. Nakamura et al. reported possible causality between chronic exposure to $\mathrm{CO}$ and cognitive dysfunction in two case reports [37]. In addition, Chen et al. used diffusion tensor imaging to demonstrate that chronic $\mathrm{CO}$ intoxication may lead to microstructural damage in corpus callosum sub-regions and cognitive impairment [38]. Previous studies have also suggested that smoking, which exposes people to the toxic constituents of tobacco smoke such as $\mathrm{CO}$, is highly correlated with an increased risk of Alzheimer's disease. Interestingly, although smoking has been associated with vascular dementia [39], some studies have reported a link between smoking and adverse effects on brain neurobiology and function in people without a history of cardiovascular disease [40]. This may suggest that CO plays a role in cognitive decline.

In this study, we found negative associations between $\mathrm{SO}_{2}$ and global cognitive decline and impairment of specific functioning domains including orientation, recall, and language, suggesting its adverse influence on neurocognitive performance. A few studies have investigated the relationships between atmospheric $\mathrm{SO}_{2}$ and neurological dysfunction [16,41]. Several studies have reported the effect of $\mathrm{SO}_{2}$ on the cardiovascular system [42,43], however few studies have reported the effect on the CNS. Hippocampal synaptic dysfunction due to $\mathrm{SO}_{2}$ exposure has been reported, and this may play a role in cognitive impairment [44]. Furthermore, co-exposure to low doses of $\mathrm{PM}_{2.5}$ and $\mathrm{SO}_{2}$ has been reported to result in neurodegeneration, including neuronal apoptosis, and decreased synaptic structural and functional proteins [45]. However, in the present study, $\mathrm{SO}_{2}$ was the one air pollutant related to most impaired trajectories of cognitive functioning according to MMSE subscales, indicating that further studies are warranted to investigate its potential damage on the CNS.

An additional important finding of this study is that high $\mathrm{NO}, \mathrm{NO}_{2}$, and $\mathrm{NO}_{\mathrm{x}}$ were associated with low total MMSE scores, and we further found that $\mathrm{NO}_{2}$ and $\mathrm{NO}_{\mathrm{x}}$ were associated with low MMSE G4 and G5 subscores. The neurotoxicity of NO as an air pollutant remains unclear. The physiological regulatory functions of endogenous NO include the modulation of blood vessel tone, immune response to neurotransmission and synaptic plasticity, and an excess of NO has been proven to be toxic [46]. In addition, the overproduction of $\mathrm{NO}$ can elicit cellular damage by causing nitrosative stress through the formation of reactive nitrogen species, and the combination of reactive nitrogen species and $\mathrm{NO}$ has been shown to be involved in the pathogenesis of neurodegenerative disorders such as Alzheimer's disease [47,48]. NO can also activate cyclooxygenase (COX), which is increased in the CNS under pro-inflammatory conditions. During its catalytic cycle, COX produces free radicals and prostaglandins, both of which can cause neurotoxicity $[49,50]$. Many studies have identified that $\mathrm{NO}_{2}$ can negatively affect cognitive function in different periods of life $[5,7,16]$. During the prenatal period, Lertxundi et al. reported that exposure to a $1 \mu \mathrm{g} / \mathrm{m}^{3}$ increase in $\mathrm{NO}_{2}$ was associated with a -0.29 point decrease in mental score in two-year-old children, which may suggest its adverse effect on cognitive development [51]. $\mathrm{NO}_{2}$ was also associated with an increased incidence of dementia in a cohort study of participants aged from 55 to 85 years [16]. As $\mathrm{NO}_{2}$ is a reactive oxidative species, the mechanism by which it damages the CNS can be assumed to involve oxidative stress, to which the brain is especially susceptible [52]. A previous animal study showed that acute $\mathrm{NO}_{2}$ exposure could induce morphological changes of the mitochondria in the cortices of rats and interfere with mitochondrial metabolism including increased generation of reactive oxygen species, which in turn affected mitochondrial energy production and biogenesis [53]. These studies may support the hypothesis that exposure to nitric compound pollutants can lead to cognitive decline.

There are several limitations to this study. First, we believe that the relationship between air pollutants and cognitive decline takes a period of time, not a short period 
of time. However, it is a cross-sectional study, and, therefore, we could not examine the effects of long-term exposure to these air pollutants on cognitive functioning. Follow-up studies are needed to confirm our results. Second, cognitive performance was assessed using only the MMSE and its subscales in our study. MMSE is a screening tool, and it only provides information about cognitive status. As a screening test, it is validated using its total score. Although different items use different cognitive functions, and some of them are summed in subscores, they are not actually sub-scales without psychometric information. Finally, levels of air pollutants were estimated at the home addresses of the participants, which is a crude measure of exposure to air pollution. Although outdoor air pollution is known to substantially contribute to indoor/personal exposure, it may not accurately reflect actual personal exposure; however, we did not have additional information about the air quality indoors.

In conclusion, the results of this study showed that exposure to different air pollutants may result in general cognitive decline and impairment of specific domains of cognitive functioning. In addition, we found that most known air pollutants are associated with cognitive decline, and this may be helpful for the government in the development of policies for early diagnosis and long-term follow-up of neurological deficits of people residing in polluted areas.

Author Contributions: Conceptualization, M.-C.C. and S.-C.C.; methodology, M.-C.C. and S.-C.C.; software, C.-F.W. and B.-C.L.; validation, S.-C.C., C.-H.H. and C.-H.K.; formal analysis, S.-W.H. and S.-C.C.; investigation, M.-C.C. and S.-C.C.; writing—original draft preparation, M.-C.C.; writingreview and editing, S.-C.C.; supervision, S.-C.C.; project administration, S.-C.C.; funding acquisition, S.-C.C. All authors have read and agreed to the published version of the manuscript.

Funding: This work was financially supported by the Research Center for Environmental Medicine, Kaohsiung Medical University, Kaohsiung, Taiwan from The Featured Areas Research Center Program within the framework of the Higher Education Sprout Project by the Ministry of Education (MOE) in Taiwan. This study was supported partially by Kaohsiung Medical University Research Center Grant (KMU-TC108A01).

Institutional Review Board Statement: The study was conducted according to the guidelines of the Declaration of Helsinki, and approved by the Institutional Review Board of Kaohsiung Medical University Chung-Ho Memorial Hospital (KMUHIRB-E(I)-20180242 and 2018/8/3 approval.

Informed Consent Statement: Informed consent was obtained from all subjects involved in the study.

Data Availability Statement: The data underlying this study is from the Taiwan Biobank. Due to restrictions placed on the data by the Personal Information Protection Act of Taiwan, the minimal data set cannot be made publicly available. Data may be available upon request to interested researchers. Please send data requests to: Szu-Chia Chen, PhD, MD. Division of Nephrology, Department of Internal Medicine, Kaohsiung Medical University Hospital, Kaohsiung Medical University.

Conflicts of Interest: The authors declare no conflict of interest.

Disclosure: We have no financial interest in the information contained in the manuscript.

\section{References}

1. North, C.M.; Rice, M.B.; Ferkol, T.; Gozal, D.; Hui, C.; Jung, S.H.; Kuribayashi, K.; McCormack, M.C.; Mishima, M.; Morimoto, Y.; et al. Air pollution in the Asia-Pacific Region: A Joint Asian Pacific Society of Respirology/American Thoracic Society perspective (Republication). Respirology 2019, 24, 484-491. [CrossRef] [PubMed]

2. Schraufnagel, D.E.; Balmes, J.R.; Cowl, C.T.; De Matteis, S.; Jung, S.H.; Mortimer, K.; Perez-Padilla, R.; Rice, M.B.; Riojas-Rodriguez, H.; Sood, A.; et al. Air Pollution and Noncommunicable Diseases: A Review by the Forum of International Respiratory Societies' Environmental Committee, Part 2: Air Pollution and Organ Systems. Chest 2019, 155, 417-426. [CrossRef] [PubMed]

3. Power, M.C.; Adar, S.D.; Yanosky, J.D.; Weuve, J. Exposure to air pollution as a potential contributor to cognitive function, cognitive decline, brain imaging, and dementia: A systematic review of epidemiologic research. Neurotoxicology 2016, 56, 235-253. [CrossRef] [PubMed]

4. Folstein, M.F.; Folstein, S.E.; McHugh, P.R. "Mini-mental state”. A practical method for grading the cognitive state of patients for the clinician. J. Psychiatr. Res. 1975, 12, 189-198. [CrossRef]

5. Kilian, J.; Kitazawa, M. The emerging risk of exposure to air pollution on cognitive decline and Alzheimer's disease-Evidence from epidemiological and animal studies. Biomed. J. 2018, 41, 141-162. [CrossRef] [PubMed] 
6. Martínez-Lazcano, J.C.; González-Guevara, E.; del Carmen Rubio, M.; Franco-Pérez, J.; Custodio, V.; Hernández-Cerón, M.; Livera, C.; Paz, C. The effects of ozone exposure and associated injury mechanisms on the central nervous system. Rev. Neurosci. 2013, 24, 337-352. [CrossRef] [PubMed]

7. Clifford, A.; Lang, L.; Chen, R.; Anstey, K.J.; Seaton, A. Exposure to air pollution and cognitive functioning across the life course-A systematic literature review. Environ. Res. 2016, 147, 383-398. [CrossRef]

8. Chang, K.H.; Chang, M.Y.; Muo, C.H.; Wu, T.N.; Chen, C.Y.; Kao, C.H. Increased risk of dementia in patients exposed to nitrogen dioxide and carbon monoxide: A population-based retrospective cohort study. PLoS ONE 2014, 9, e103078. [CrossRef]

9. Choe, Y.M.; Lee, B.C.; Choi, I.G.; Suh, G.H.; Lee, D.Y.; Kim, J.W. Alzheimer's Disease Neuroimaging Initiative. MMSE Subscale Scores as Useful Predictors of AD Conversion in Mild Cognitive Impairment. Neuropsychiatr. Dis. Treat. 2020, 16, $1767-1775$. [CrossRef]

10. Chen, C.H.; Yang, J.H.; Chiang, C.W.K.; Hsiung, C.N.; Wu, P.E.; Chang, L.C.; Chu, H.W.; Chang, J.; Song, I.W.; Yang, S.L.; et al. Population structure of Han Chinese in the modern Taiwanese population based on 10,000 participants in the Taiwan Biobank project. Hum. Mol. Genet. 2016, 25, 5321-5331. [CrossRef] [PubMed]

11. Fan, C.T.; Hung, T.H.; Yeh, C.K. Taiwan Regulation of Biobanks. J. Law Med. Ethics 2015, 43, 816-826. [PubMed]

12. Levey, A.S.; Bosch, J.P.; Lewis, J.B.; Greene, T.; Rogers, N.; Roth, D. A more accurate method to estimate glomerular filtration rate from serum creatinine: A new prediction equation. Modification of Diet in Renal Disease Study Group. Ann. Intern. Med. 1999, 130, 461-470. [CrossRef] [PubMed]

13. Lin, Y.H.; Wang, C.F.; Chiu, H.; Lai, B.C.; Tu, H.P.; Wu, P.Y.; Huang, J.C.; Chen, S.C. Air Pollutants Interaction and Gender Difference on Bone Mineral Density T-Score in Taiwanese Adults. Int. J. Environ. Res. Public Health 2020, 17, 9165. [CrossRef] [PubMed]

14. Lo, Y.C.; Lu, Y.C.; Chang, Y.H.; Kao, S.; Huang, H.B. Air Pollution Exposure and Cognitive Function in Taiwanese Older Adults: A Repeated Measurement Study. Int. J. Environ. Res. Public Health 2019, 16, 2976. [CrossRef]

15. Jung, C.-R.; Lin, Y.-T.; Hwang, B.-F. Ozone, particulate matter, and newly diagnosed Alzheimer's disease: A population-based cohort study in Taiwan. J. Alzheimer's Dis. 2015, 44, 573-584. [CrossRef] [PubMed]

16. Chen, H.; Kwong, J.C.; Copes, R.; Hystad, P.; van Donkelaar, A.; Tu, K.; Brook, J.R.; Goldberg, M.S.; Martin, R.V.; Murray, B.J. Exposure to ambient air pollution and the incidence of dementia: A population-based cohort study. Environ. Int. 2017, 108, 271-277. [CrossRef] [PubMed]

17. Shin, J.; Han, S.-H.; Choi, J. Exposure to Ambient Air Pollution and Cognitive Impairment in Community-Dwelling Older Adults: The Korean Frailty and Aging Cohort Study. Int. J. Environ. Res. Public Health 2019, 16, 3767. [CrossRef]

18. Wang, J.; Li, T.; Lv, Y.; Kraus, V.B.; Zhang, Y.; Mao, C.; Yin, Z.; Shi, W.; Zhou, J.; Zheng, T.; et al. Fine Particulate Matter and Poor Cognitive Function among Chinese Older Adults: Evidence from a Community-Based, 12-Year Prospective Cohort Study. Environ. Health Perspect. 2020, 128, 67013. [CrossRef] [PubMed]

19. Calderón-Garcidueñas, L.; Mukherjee, P.S.; Kulesza, R.J.; Torres-Jardón, R.; Hernández-Luna, J.; Ávila-Cervantes, R.; MacíasEscobedo, E.; González-González, O.; González-Maciel, A.; García-Hernández, K.; et al. Mild Cognitive Impairment and Dementia Involving Multiple Cognitive Domains in Mexican Urbanites. J. Alzheimers Dis. 2019, 68, 1113-1123.

20. Pun, V.C.; Manjourides, J.; Suh, H. Association of ambient air pollution with depressive and anxiety symptoms in older adults: Results from the NSHAP study. Environ. Health Perspect. 2017, 125, 342-348. [CrossRef]

21. Liu, X.-B.; Shen, Y.; Plane, J.M.; Deng, W. Vulnerability of premyelinating oligodendrocytes to white-matter damage in neonatal brain injury. Neurosci. Bull. 2013, 29, 229-238. [CrossRef] [PubMed]

22. Wang, Y.; Xiong, L.; Tang, M. Toxicity of inhaled particulate matter on the central nervous system: Neuroinflammation, neuropsychological effects and neurodegenerative disease. J. Appl. Toxicol. 2017, 37, 644-667. [CrossRef] [PubMed]

23. Bencsik, A.; Lestaevel, P.; Guseva Canu, I. Nano- and neurotoxicology: An emerging discipline. Prog. Neurobiol. 2018, 160, 45-63. [CrossRef] [PubMed]

24. Shou, Y.; Huang, Y.; Zhu, X.; Liu, C.; Hu, Y.; Wang, H. A review of the possible associations between ambient PM2. 5 exposures and the development of Alzheimer's disease. Ecotoxicol. Environ. Saf. 2019, 174, 344-352. [CrossRef]

25. Cacciottolo, M.; Wang, X.; Driscoll, I.; Woodward, N.; Saffari, A.; Reyes, J.; Serre, M.L.; Vizuete, W.; Sioutas, C.; Morgan, T.E.; et al. Particulate air pollutants, APOE alleles and their contributions to cognitive impairment in older women and to amyloidogenesis in experimental models. Transl. Psychiatry 2017, 7, e1022. [CrossRef]

26. Bhatt, D.P.; Puig, K.L.; Gorr, M.W.; Wold, L.E.; Combs, C.K. A pilot study to assess effects of long-term inhalation of airborne particulate matter on early Alzheimer-like changes in the mouse brain. PLoS ONE 2015, 10, e0127102. [CrossRef]

27. Hedges, D.W.; Erickson, L.D.; Kunzelman, J.; Brown, B.L.; Gale, S.D. Association between exposure to air pollution and hippocampal volume in adults in the UK Biobank. Neurotoxicology 2019, 74, 108-120. [CrossRef]

28. Chen, J.-C.; Schwartz, J. Neurobehavioral effects of ambient air pollution on cognitive performance in US adults. Neurotoxicology 2009, 30, 231-239. [CrossRef]

29. Tonne, C.; Elbaz, A.; Beevers, S.; Singh-Manoux, A. Traffic-related air pollution in relation to cognitive function in older adults. Epidemiology 2014, 25, 674-681. [CrossRef]

30. Schikowski, T.; Vossoughi, M.; Vierkötter, A.; Schulte, T.; Teichert, T.; Sugiri, D.; Fehsel, K.; Tzivian, L.; Bae, I.S.; Ranft, U.; et al. Association of air pollution with cognitive functions and its modification by APOE gene variants in elderly women. Environ. Res. 2015, 142, 10-16. [CrossRef] 
31. Gatto, N.M.; Henderson, V.W.; Hodis, H.N.; St John, J.A.; Lurmann, F.; Chen, J.C.; Mack, W.J. Components of air pollution and cognitive function in middle-aged and older adults in Los Angeles. Neurotoxicology 2014, 40, 1-7. [CrossRef] [PubMed]

32. Cleary, E.G.; Cifuentes, M.; Grinstein, G.; Brugge, D.; Shea, T.B. Association of low-level ozone with cognitive decline in older adults. J. Alzheimer's Dis. 2018, 61, 67-78. [CrossRef] [PubMed]

33. Carey, I.M.; Anderson, H.R.; Atkinson, R.W.; Beevers, S.D.; Cook, D.G.; Strachan, D.P.; Dajnak, D.; Gulliver, J.; Kelly, F.J. Are noise and air pollution related to the incidence of dementia? A cohort study in London, England. BMJ Open 2018, 8, e022404. [CrossRef] [PubMed]

34. Galiè, M.; Covi, V.; Tabaracci, G.; Malatesta, M. The role of Nrf2 in the antioxidant cellular response to medical ozone exposure. Int. J. Mol. Sci. 2019, 20, 4009. [CrossRef] [PubMed]

35. Doyle, K.P.; Simon, R.P.; Stenzel-Poore, M.P. Mechanisms of ischemic brain damage. Neuropharmacology 2008, 55, 310-318. [CrossRef] [PubMed]

36. Rose, J.J.; Wang, L.; Xu, Q.; McTiernan, C.F.; Shiva, S.; Tejero, J.; Gladwin, M.T. Carbon Monoxide Poisoning: Pathogenesis, Management, and Future Directions of Therapy. Am. J. Respir. Crit. Care Med. 2017, 195, 596-606. [CrossRef]

37. Nakamura, T.; Setsu, K.; Takahashi, T.; Miyashita, M.; Sugiyama, N.; Washizuka, S.; Murata, S.; Hanihara, T.; Amano, N. Chronic exposure to carbon monoxide in two elderly patients using a kotatsu, a traditional Japanese charcoal-based heater. Psychogeriatrics 2016, 16, 323-326. [CrossRef]

38. Chen, P.C.; Chen, M.H.; Chen, H.L.; Lu, C.H.; Chou, K.H.; Wu, R.W.; Tsai, N.W.; Lin, C.P.; Li, S.H.; Chen, Y.W.; et al. Callosal damage and cognitive deficits in chronic carbon monoxide intoxication: A diffusion tensor imaging study. J. Neurol. Sci. 2015, 355, 101-107. [CrossRef]

39. Hersi, M.; Irvine, B.; Gupta, P.; Gomes, J.; Birkett, N.; Krewski, D. Risk factors associated with the onset and progression of Alzheimer's disease: A systematic review of the evidence. Neurotoxicology 2017, 61, 143-187. [CrossRef]

40. Durazzo, T.C.; Meyerhoff, D.J.; Nixon, S.J. Chronic cigarette smoking: Implications for neurocognition and brain neurobiology. Int. J. Environ. Res. Public Health 2010, 7, 3760-3791. [CrossRef]

41. Gui, Z.; Cai, L.; Zhang, J.; Zeng, X.; Lai, L.; Lv, Y.; Huang, C.; Chen, Y. Exposure to ambient air pollution and executive function among Chinese primary schoolchildren. Int. J. Hyg. Environ. Health 2020, 229, 113583. [CrossRef] [PubMed]

42. Ab Manan, N.; Noor Aizuddin, A.; Hod, R. Effect of Air Pollution and Hospital Admission: A Systematic Review. Ann. Glob. Health 2018, 84, 670-678. [CrossRef] [PubMed]

43. Yu, W.; Jin, H.; Tang, C.; Du, J.; Zhang, Z. Sulfur-containing gaseous signal molecules, ion channels and cardiovascular diseases. Br. J. Pharmacol. 2018, 175, 1114-1125. [CrossRef] [PubMed]

44. Yun, Y.; Yao, G.; Yue, H.; Guo, L.; Qin, G.; Li, G.; Sang, N. SO 2 inhalation causes synaptic injury in rat hippocampus via its derivatives in vivo. Chemosphere 2013, 93, 2426-2432. [CrossRef] [PubMed]

45. Ku, T.; Chen, M.; Li, B.; Yun, Y.; Li, G.; Sang, N. Synergistic effects of particulate matter $\left(\mathrm{PM}_{2.5}\right)$ and sulfur dioxide $\left(\mathrm{SO}_{2}\right)$ on neurodegeneration via the microRNA-mediated regulation of tau phosphorylation. Toxicol. Res. 2017, 6, 7-16. [CrossRef]

46. Pacher, P.; Beckman, J.S.; Liaudet, L. Nitric oxide and peroxynitrite in health and disease. Physiol. Rev. 2007, 87, 315-424. [CrossRef]

47. Sultana, R.; Poon, H.F.; Cai, J.; Pierce, W.M.; Merchant, M.; Klein, J.B.; Markesbery, W.R.; Butterfield, D.A. Identification of nitrated proteins in Alzheimer's disease brain using a redox proteomics approach. Neurobiol. Dis. 2006, 22, 76-87. [CrossRef]

48. Guix, F.; Uribesalgo, I.; Coma, M.; Munoz, F. The physiology and pathophysiology of nitric oxide in the brain. Prog. Neurobiol. 2005, 76, 126-152. [CrossRef]

49. Mancuso, C.; Scapagini, G.; Curro, D.; Giuffrida Stella, A.M.; De Marco, C.; Butterfield, D.A.; Calabrese, V. Mitochondrial dysfunction, free radical generation and cellular stress response in neurodegenerative disorders. Front. Biosci. 2007, 12, 1107-1123. [CrossRef]

50. Calabrese, V.; Mancuso, C.; Calvani, M.; Rizzarelli, E.; Butterfield, D.A.; Stella, A.M.G. Nitric oxide in the central nervous system: Neuroprotection versus neurotoxicity. Nat. Rev. Neurosci. 2007, 8, 766-775. [CrossRef]

51. Lertxundi, A.; Baccini, M.; Lertxundi, N.; Fano, E.; Aranbarri, A.; Martínez, M.D.; Ayerdi, M.; Álvarez, J.; Santa-Marina, L.; Dorronsoro, M.; et al. Exposure to fine particle matter, nitrogen dioxide and benzene during pregnancy and cognitive and psychomotor developments in children at 15 months of age. Environ. Int. 2015, 80, 33-40. [CrossRef] [PubMed]

52. Mattson, M.P. Mechanisms of Neuronal Apoptosis and Excitotoxicity. In Pathogenesis of Neurodegenerative Disorders; Springer: New York, NY, USA, 2001; pp. 1-20.

53. Yan, W.; Ji, X.; Shi, J.; Li, G.; Sang, N. Acute nitrogen dioxide inhalation induces mitochondrial dysfunction in rat brain. Environ. Res. 2015, 138, 416-424. [CrossRef] [PubMed] 